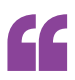

cognitive impairment was associated with reduced white matter integrity in [remote] regions

STROKE

\title{
Remote white matter integrity influences cognitive function after stroke
}

Cognitive impairment after ischaemic stroke in young adults could be attributable to white matter damage in brain regions distant from the original lesion site, according to new research published in Stroke. These findings might help to explain why cognitive outcomes do not always reflect stroke severity.

"An ischaemic stroke is a focal event, but cognitive impairment cannot always be explained by the lesion characteristics," says Frank-Erik de Leeuw, who led the new study. "This phenomenon is nicely illustrated by our previous work, which found frequent occurrence of episodic memory impairment after stroke, in the absence of lesions in brain structures known to be important for memory formation, such as the medial temporal lobe."

The research was conducted as part of the FUTURE study, an ongoing prospective cohort study that is investigating stroke risk factors and prognosis in younger individuals. The participants were drawn from a registry of patients admitted to the
Radboud University Medical Center in the Netherlands between 1980 and 2010.

The new study included 117 patients aged $18-50$ years with firsttime ischaemic stroke, along with 84 stroke-free controls matched for age, sex and education. The patients were assessed 10.7 years, on average, after stroke. T1-weighted MRI and diffusion tensor imaging were used to measure white matter integrity, and the participants also underwent neuropsychological assessments.

The researchers found that cognitive impairment was associated with reduced white matter integrity in regions remote from the original lesion, including the contralesional hemisphere in some cases. By contrast, the remote white matter remained relatively intact in individuals who did not develop cognitive impairment after stroke.

The mechanisms that underlie the effects of stroke on white matter distant from the lesion site are unclear. Stroke volume was found to correlate with loss of white matter integrity, but did not seem to be the only relevant factor, as the relationship between white matter compromise and cognitive impairment persisted after adjustment for lesion volume.

"Future research should use a longitudinal design, which can provide valuable insights into changes in cognitive function over time after stroke, as well as their relationship with the course of remote white matter integrity changes," comments de Leeuw. The researchers also hope to find out whether stroke patients who are at risk of remote structural changes can be identified at an early stage, so that their poststroke management can be tailored accordingly.

Heather Wood

ORIGINAL ARTICLE Schnaapsmeerders, P. et al. Remote lower white matter integrity increases the risk of long-term cognitive impairment after ischemic stroke in young adults. Stroke http:// dx.doi.org/10.1161/STROKEAHA.116.014356 (2016)

FURTHER READING Rutten-Jacobs, L. C. et al. Risk factors and prognosis of young stroke. The FUTURE study: a prospective cohort study. Study rationale and protocol. BMC Neurol. 11, 109 (2011) 